\title{
EuroSCORE II, is its predictive capacity influenced by the patient's risk profile?
}

\author{
Jesus Herreros ${ }^{1,2}$, Juan Bustamante-Munguira ${ }^{3}$ \\ ${ }^{1}$ Unidad de Cirugía Cardiaca, Hospital Nisa Pardo Arvaca, Madrid, Spain \\ ${ }^{2}$ Cátedra de Ingeniería Biomédica y tecnologías Sanitarias, \\ Universidad Católica San Antonio (UCAM), Murcia, Spain \\ ${ }^{3}$ Servicio de Cirugía Cardiovascular, Hospital Universitario de La Princesa, Madrid, Spain
}

\section{Article p. 495}

Methodology in the stratification of surgical risk allows us to design predictive models based on the patient's variables that will allow us to stratify them according to surgical risk. The additive EuroSCORE risk model was developed based on 19,030 patients from 128 hospitals in eight European countries who underwent cardiac surgery between September and November 1995 [1]. Ninety-seven risk factors were studied and, of those, 17 were identified as being significantly associated with increased surgical mortality. The additive EuroSCORE overpredicts risk in coronary and valvular surgery, underpredicts observed mortality for combined valve and coronary surgery and has poor predictive ability for patients at higher risk [2].

The logistic EuroSCORE, published in 2003, uses a more complex algorithm to be a better predictor than the additive model, using the same preoperative risk factors [3]. It has been shown to be a better predictor than the additive EuroSCORE for high-risk patients, but continues to overestimate mortality by a factor of approximately 2.5 in the 6 risk classes in European, American and Australian studies [2, 4]. These results have been associated to improvements in surgical techniques and perioperative care which results in a reduction of hospital mortality. These results reinforce the hypothesis that patients who would have died one or two decades ago, currently survive thanks to improved perioperative care.

The update to EuroSCORE II is based on 23,000 patients having undergone cardiac surgery in 150 hospitals in 43 countries between May and July 2010 [5]. EuroSCORE II has improved risk prediction in combined aortic valve replacement and high-risk patients. However, it is poorly calibrated in the lowest-risk patients. In isolated coronary bypass surgery, it has been published that the original EuroSCORE has a better fit than EuroSCORE II, raising concerns over its replacement $[6,7]$.

Nevertheless, the EuroSCORE and other risk models have certain limitations in terms of the stratification of surgical risk:

- The result, or dependent variable, is hospital mortality. Other relevant aspects, such as predicting complications during surgery, the duration of post-operative hospital stay, perceived quality by the patient, functional class and quality of life, are not measured as dependent variables. The EuroSCORE observes only a short and possibly biased interval. The wrong or incomplete therapeutic process could be associated with differences in short- and long-term outcomes. The time-spans in the evaluation of outcomes are often under measured.

- The majority of the risk stratification models are static. The information was obtained in a determinant moment, the model was developed and then validated $a$ posteriori. This can lead to the overestimation of risk using EuroSCORE. One of the advantages of The National Database of the Society of Thoracic Surgeons is that it is updated annually, and from the operative point of view, it is the optimal system.

Address for correspondence: Jesus Herreros, MD, PhD, Unidad de Cirugía Cardiaca, Hospital Nisa Pardo Arvaca, Madrid, Spain; Cátedra de Ingeniería Biomédica y tecnologías Sanitarias, Universidad Católica San Antonio (UCAM), Murcia, Spain, e-mail: jesus.herreros@gmail.com 
- The potential part of subjectivity in the encoding method inside the EuroSCORE and its possible impact on the score's predictive power has been investigated. The inter-observer discrepancies, between surgeon and perfusionist were observed in $26 \%$ of patients [8]. As surgeon prediction value was more often superior to perfusionist value than the reverse, one may hypothesize that surgeon attempted to game the scoring system. Factors relevant to EuroSCORE (ejection fraction, serum creatinine levels and pulmonary systolic pressure) may vary as a result of medical preoperative treatment. Any of these variables may also vary according to which imaging tests are used to measure for example the ejection fraction (echocardiography vs. angiography). Some definitions may still give room for error by interpretation. Another aspect is the codification of the procedure. In the case of aortic valve replacement, where an aortic patch is used to enlarge the aortic annulus or the aortotomy, is it to be considered surgery of the thoracic aorta? There is some disagreement among observers about the scoring of unstable and recent myocardial infarction, probably as a result of changes in the international definition of myocardial infarction and unstable angina in the new overall concept of acute coronary syndromes.

- Risk factors that are known to affect mortality, such as hepatic cirrhosis or mediastinal radiotherapy, are not included in the dataset. Medical conditions affecting mobility are included in EuroSCORE II under the factor "poor mobility" [6]. However, medical conditions that do not affect mobility are not included. Anatomical features like the degree of aortic calcification or an incomplete revascularization neither are included. The SYNTAX study (synergy between percutaneous coronary intervention with Taxus and cardiac surgery), includes a score based on the characteristics of the coronary lesions, such as calcification, total occlusion and coronary arteries of less than $2.5 \mathrm{~mm}$ [9].

- EuroSCORE has been used to compare conventional surgery with alternative procedures, like percutaneous coronary interventions or trans-aortic valve implantation [10]. Overprediction of mortality and the absence of long-term evaluation can lead to mistaken decisions to opt for alternative procedures. In the past, the use of a score for the evaluation of the practices in cardiac surgery had an impact on how users encoded. The most publicized example was the logistic model of the New York State in the 1990s, which published the ranking of cardiac surgeons in New York Times. A shift in patient risk profile was observed for some surgeons, who improved by the way, their ranking [8]. Risk stratification is essential for the monitoring of surgeons and hospitals. Reliance on a risk stratification system that over-predicts mortality may have serious consequences. It leads to a false sense of reassurance, underperformance may be undetected and patient welfare may be compromised [2]. On the other hand, surgeons may reject the indication of surgery in high-risk patients because of factors not included in the EuroSCORE dataset.

In this issue of "Cardiology Journal", the Hospital de Clínicas of Buenos Aires group [11] publishes a validation of EuroSCORE II in low- and verylow-risk patients. A validation of EuroSCORE II in this group of patients is very interesting because it has been less studied than in high-risk patients. In the group of patients with low mortality, in order to be able to establish a validation, it is necessary to have a very large sample. Furthermore, this group tends to be patients with ischemic cardiopathy. The authors conclude that this risk model proved to be useful in evaluating the quality standards of local cardiac surgery, and that the review of cases of deaths provides valuable information for improving quality of care. Precision in the prediction of surgical risk is a tool which, though it may not offer results in the medium- to long-term, and does not take into account the anatomic characteristics of the coronary lesion, may, nevertheless, aid better therapeutic decision-making when deciding between coronary surgery and percutaneous coronary intervention.

\section{Conflict of interest: None declared}

\section{References}

1. Nashef SA, Roques F, Michel P, Gauducheau E, Lemeshow S, Salamon R. European system for cardiac operative risk evaluation (EuroSCORE). Eur J Cardiothorac Surg, 1999; 16: 9-13.

2. Choong CK, Sergeant P, Nashef SAM, Smith JA, Bridgewater B. The EuroSCORE risk stratification system in the current era: how accurate is it and what should be done if it is inaccurate? (Editorial). Eur J Cardiotorac Surg, 2009; 35: 54-61.

3. Roques F, Michel P, Goldstone AR, Nashed SA. The logistic EuroSCORE. Eur Heart J, 2003; 24: 881-882.

4. Bhatti F, Grayson AD, Grotte G et al. The logistic EuroSCORE in cardiac surgery: how well does it predict operative risk? Heart, 2006; 92: 1817-1820.

5. Nashef SA, Roques F, Sharples LD et al. EuroSCORE II. Eur J Cardiothorac Surg, 2012; 41: 734-744. 
6. Chalmers J, Pullan M, Fabri B et al. Validaton of EuroSCORE II in a modern cohort of patients undergoing cardiac surgery. Eur J Cardiothorac Surg, 2013; 43: 684-694.

7. Borracci RA, Rubio M, Celano L, Ingino CA, Allende NG, Guerrero RAA. Prospective validation of EuroSCORE II in patients undergoing cardiac surgery in Argentinean centres. Interact CardioVasc Thorac Surg, 2014; 18: 539-543.

8. Lebreton $\mathrm{G}$, Merle $\mathrm{S}$, Inamo $\mathrm{J}$ et al. Limitations in the interobservr reliability of EuroSCORE: what should change in EuroSCORE II?. Eur J Cardiothorac Surg, 2011; 40: 1304-1308.

9. Mohr FW, Morice MC, Kappetein AP et al. Coronary artery bypas graft surgery versus percutaneous intervention in patients with three-vessel disease and left main coronary disease: 5 year follow-up on the randomised, clinical SYNTAX trial. Lancet, 2013; 381: 629-638.

10. Himbert D, Descoutures F, Al-Attar N et al. Results of transfemoral or transapical aortic valve implantation following a uniform assessment in high-risk patients with aortic stenosis. J Am Coll Cardiol, 2009; 54: 303-311.

11. Borracci RA, Rubio M, Baldi J, Jr., Ahuad Guerrero RA, Mauro V. Mortality in low- and very low-risk patients undergoing cardiac surgery: EuroSCORE II as a new standard. Cardiol J, 2015; 22: 495-500. 